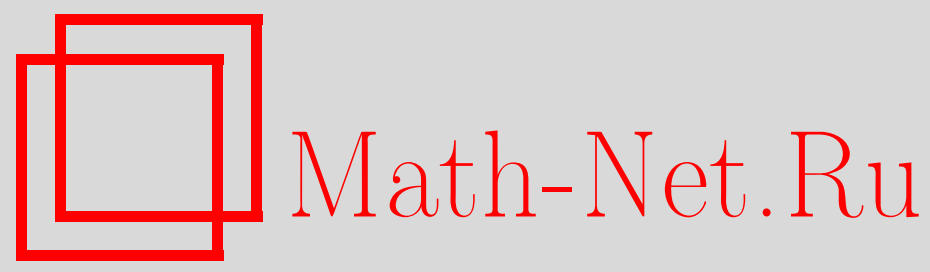

Г. Г. Амосов, А. Д. Баранов, О дилатации сжимающих коциклов и коциклических возмущениях группы сдвигов на прямой, Матем. заметки, 2006, том 79, выпуск 1, 3-18

DOI: https://doi.org/10.4213/mzm2669

Использование Общероссийского математического портала Math-Net.Ru подразумевает, что вы прочитали и согласны с пользовательским соглашением http://www . mathnet.ru/rus/agreement

Параметры загрузки:

IP : 54.198 .187 .58

26 апреля 2023 г., 04:06:42

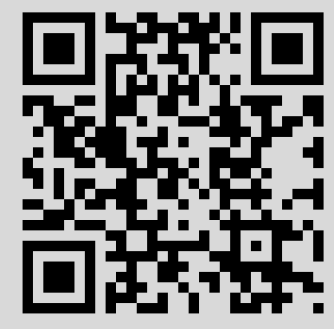




\title{
О ДИЛАТАЦИИ СЖИМАЮЩИХ КОЦИКЛОВ И КОЦИКЛИЧЕСКИХ ВОЗМУЩЕНИЯХ ГРУППЫ СДВИГОВ НА ПРЯМОЙ
}

\author{
Г. Г. Амосов, А. Д. Баранов
}

\begin{abstract}
Введен класс сжимающих коциклов, для которых существуют дилатации до унитарных марковских коциклов группы сдвигов на прямой $S$. Исследован класс коцик лических возмущений $S$ унитарными марковскими коциклами $W$ со свойством $W_{t}-I \in \mathscr{S}_{2}$ (класс Гильберта-Шмидта). Полученные результаты применяются к задаче о возмущении колмогоровских потоков на гиперфинитных факторах, порожденных алгеброй канонических антикоммутационных соотношений.
\end{abstract}

Библиография: 24 названия.

1. Введение. Пусть $U=\left(U_{t}\right)_{t \in \mathbb{R}}$ есть $C_{0}$-группа унитарных операторов в гильбертовом пространстве $H$. Формула $x \rightarrow U_{t} x U_{-t}, x \in B(H), t \in \mathbb{R}$, определяет естественное действие группы $U$ на алгебре всех ограниченных операторов $B(H)$ в гильбертовом пространстве $H$. Заметим, что семейство $\alpha_{t}(\cdot)=U_{t} \cdot U_{-t}, t \in \mathbb{R}$, образует групшу автоморфизмов алгебры $B(H)$. Сильно непрерьвное семейство сжимающих операторов $L=\left(L_{t}\right)_{t \geqslant 0}$ называется (сжимающим) коциклом грушы $U$, если вьполнено условие

$$
L_{t+s}=L_{t} \alpha_{t}\left(L_{s}\right), \quad s, t \geqslant 0 .
$$

Условие (1) в точности означает, что семейство $T_{t}=L_{t} U_{t}, t \geqslant 0$, образует $C_{0}$-полугруппу сжатий $T$, которую мы будем называть кочиклическим возмущениемгрупы $U$. В том случае, когда коцикл $L$ состоит из унитарных операторов, он единственным образом доопределяется для отрицательных значений параметра $t$ по формуле $L_{-t}=\alpha_{t}\left(L_{t}^{*}\right)$, $t \geqslant 0$. Получившееся семейство будет удовлетворять условию (1) для всех значений параметров $t, s \in \mathbb{R}$. В этом случае, коциклическое возмущение $T$ продолжается до группы унитарных операторов. Заметим, что если $U_{t}=\mathrm{Id}, t \in \mathbb{R}$, то сжимаюший коцикл $L$ образует $C_{0}$-полугрупу, т.е. $L_{t+s}=L_{t} L_{s}, t, s \geqslant 0$.

Унитарный коцикл $W$ группы унитарных операторов $\widetilde{S}$ в гильбертовом пространстве

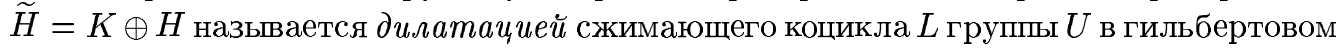
пространстве $K$, если

$$
L_{t}=\left.P_{K} W_{t}\right|_{K}, \quad t \geqslant 0,
$$

где $P_{K}$ есть ортогональньй проектор в $\widetilde{H}$ на подпространство $K$, а $\widetilde{S}=U \oplus S$ для некоторой групшы унитарных операторов $S$ в гильбертовом пространстве $H$. Дилатация

Работа выполнена при частичной поддержке программы "Ведущие научные школы", грант № HШ-2266.2003.1. 
назьвается минимальной, если замыкание линейной оболочки множества $\left\{W_{t} \eta, \eta \in K\right.$, $t \geqslant 0\}$ совпадает с $\widetilde{H}$.

Пусть $S$ есть группа сдвигов в гильбертовом пространстве $H=L^{2}(\mathbb{R})$, определенная формулой $\left(S_{t} \eta\right)(x)=\eta(x-t), \eta \in H$. Монотонно возрастающее при возрастании $t$ семейство подпространств $H_{t} \subset H$, состоящих из функций с носителем на луче $[-t,+\infty)$, образует естественную фильтрацию пространства $H$. Унитарньй коцикл $W$ группы сдвигов $S$ называется марковским [1]-[4], если $\left.W_{t}\right|_{H_{0}^{\perp}}=\mathrm{Id}, t \geqslant 0$. Пусть группа унитарных операторов $U$ действует в гильбертовом пространстве $K$. Мы будем говорить, что коцикл $W$ группы $\widetilde{S}=U \oplus S$, действующий в гильбертовом пространстве $\widetilde{H}=K \oplus H$, марковский, если $\left.W_{t}\right|_{\left(K \oplus H_{0}\right)^{\perp}}=\mathrm{Id}, t \geqslant 0$. С точки зрения квантовой теории открытых систем пространство $K$ имеет смысл системы (одночастичной) с шумовым окружением $H$. Термин "марковский коцикл” традиционно используется для коциклов в пространстве Фока $\Gamma(\widetilde{H}) \approx \Gamma(K) \otimes \Gamma(H)$, согласованньх с естественной фильтрацией пространства $H$. Теория таких коциклов широко используется в квантовом стохастическом исчислении (см. [5]-[8]). Мы называем “марковскими" коциклы в одночастичном пространстве $K \oplus H$, вторичное квантование которых приводит к марковским коциклам в смысле общепринятого определения.

В работе [9] рассматривалась задача о дилатации сжимающих коциклов до изометрических для случая коциклов полугрупп эндоморфизмов. Это явилось некоторьм стимулом для изучения нами дилатаций коциклов в первой части нашей работы.

Во второй части работы мы рассматриваем задачу о построении коциклов $W$, удовлетворяющих условию $W_{t}-I \in \mathscr{S}_{2}$. Такая задача естественным образом появляется при исследовании коциклических возмущений на гиперфинитных факторах фон Неймана, поскольку указанное условие должно вьполняться для того, чтобы можно было корректно определить вторичное квантование коциклов $W$ (см. [10]). Заметим, что (см. [11]) вторичное квантование групш сдвигов приводит к колмогоровским потокам на гиперфинитных факторах фон Неймана, канонически связанным с потоками сдвигов Пауэрса [12]. Исследования коциклических возмущений потоков сдвигов Пауэрса инициированы в [13] и проводятся, в последнее время, очень интенсивно (см. [14], [15]).

Предлагаемая работа построена следующим образом. В разделе 2 мы показываем, в каком смысле единственна минимальная дилатация коцикла. Далее, мы вводим модель марковского коцикла группы сдвигов и, с ее помощью, доказываем существование дилатации для случая, когда коциклическое возмущение является группой “усеченных сдвигов" . В разделе 3 мы рассматриваем возмущения групш сдвигов на прямой унитарными марковскими коциклами $W$ и формулируем утверждение, позволяющее описать широкий класс возмущений коциклами, удовлетворяющими дополнительному условию $W_{t}-I \in \mathscr{S}_{2}$. Доказательство этого утверждения существенно опирается на теорему о триангулящии полугрупы усеченных сдвигов, доказательство которой приведено в разделе 4. Раздел 5 посвящен доказательству утверждения, сформулированного в третьей части. Наконец, в разделе 6 мы демонстрируем некоторые приложения полученных нами результатов к изучению коциклических возмущений колмогоровских потоков гиперфинитных факторов, порожденных алгеброй канонических антикоммутационных соотношений (KАС). В приложении приведены основные сведения об алгебре КАС. Мы предполагаем, что полученные нами результаты имеют приложения не только для квазисвободных эволюций алгебры КАС, но и для других гиперфинитных алгебр фон Неймана, например, для алгебр, порожденных представлениями алгебры квадрата квантового белого шума (см. [16]). 
2. Модель унитарного марковского коцикла группы сдвигов. Существование и единственность дилатации. Следующие два утверждения дают представление о степени единственности дилатации сжимающего коцикла.

ПРЕДЛОЖЕНИЕ 1. Предположиим, что $W$ и $W^{\prime}$ есть две минимальнье дилатаиии сәсимающего коцикла $L$ группы $U$ до унитарных кочиклов групп $\widetilde{S}=U \oplus S u$ $\widetilde{S}^{\prime}=U \oplus S^{\prime}$ соответственно, причем

$$
W_{t} \eta=W_{t}^{\prime} \eta, \quad \eta \in K, \quad t \geqslant 0
$$

Тогда коциклические возмущения групп $\widetilde{S}$ и $\widetilde{S}^{\prime}$ коциклами $W$ и $W^{\prime}$ совпадают, так чmo $W_{t} \widetilde{S}_{t}=W_{t}^{\prime} \widetilde{S}_{t}^{\prime}, t \in \mathbb{R}$.

ДокАЗАТЕЛЬСТво. Заметим, что из условия $W_{t} \eta=W_{t}^{\prime} \eta, \eta \in K$, следует

$$
W_{t} \widetilde{S}_{t} \eta=W_{t}^{\prime} \widetilde{S}_{t} \eta=W_{t}^{\prime} \widetilde{S}_{t}^{\prime} \eta, \quad \eta \in K
$$

поскольку подпространство $K$ приводит группы $\widetilde{S}$ и $\widetilde{S}^{\prime}$, причем $\left.\widetilde{S}\right|_{K}=\left.\widetilde{S}^{\prime}\right|_{K^{\prime}}=U$. Из $_{3}$ условия коцикла следует, что семейства операторов $V_{t}^{(1)}=W_{t} \widetilde{S}_{t}$ и $V_{t}^{(2)}=W_{t}^{\prime} \widetilde{S}_{t}, t \in \mathbb{R}$, образуют группы унитарных операторов в $\widetilde{H}$. В силу (2) мы получаем

$$
\begin{aligned}
V_{t}^{(1)} W_{s} \eta & =V_{t}^{(1)} W_{s} \widetilde{S}_{s} \widetilde{S}_{-s} \eta=V_{t}^{(1)} V_{s}^{(1)} \widetilde{S}_{-s} \eta \\
& =V_{t+s}^{(1)} \widetilde{S}_{-s}^{\prime} \eta=V_{t+s}^{(2)} \widetilde{S}_{-s}^{\prime} \eta=V_{t}^{(2)} W_{s}^{\prime} \eta .
\end{aligned}
$$

Мы использовали здесь свойство $\widetilde{S}_{-s} \eta=U_{-t} \eta=\widetilde{S}_{-s}^{\prime} \eta \in K$. В силу минимальности дилатации из равенства (3) следует, что $V_{t}^{(1)}=V_{t}^{(2)} \equiv V_{t}, t \geqslant 0$.

СледСТВИЕ. Предположим, что $W$ и $W^{\prime}$ есть две минимальные дилатации сәсимающего коцикла $L$ группь $U$ до унитарных коциклов одной и той же әруппь $\widetilde{S}=U \oplus S$, причем

$$
W_{t} \eta=W_{t}^{\prime} \eta, \quad \eta \in K, \quad t \geqslant 0
$$

Тогда

$$
W_{t}=W_{t}^{\prime}, \quad t \in \mathbb{R}
$$

ДокАЗАТЕльСТво. Согласно предложению $1 W_{t} \widetilde{S}_{t}=W_{t}^{\prime} \widetilde{S}_{t} \equiv V_{t}, t \in \mathbb{R}$, откуда $W_{t}^{\prime}=V_{t} \widetilde{S}_{-t}=W_{t}, t \in \mathbb{R}$.

Займемся теперь исследованием существования дилатации. Подпространство $H_{0}=$ $L^{2}\left(\mathbb{R}_{+}\right)$инвариантно относительно операторов сдвига $S_{t}$ при $t \geqslant 0$. Следовательно, семейство операторов $\left.S_{t}\right|_{H_{0}}, t \geqslant 0$, образует полугрупшу изометрических операторов, назьваемую полугруппй сдвигов. Мы будем обозначать операторы, принадлежащие этой полугруппе, тем же символом, что операторы групшы сдвигов в $H$. Согласно классической теореме Лакса (см. [17, лекция XI]) подпространство $E \subset H_{0}$ инвариантно относительно полугрупшы сдвигов, $S_{t} E \subset E, t \geqslant 0$, тогда и только тогда, когда найдется внутренняя в верхней полуплоскости $\mathbb{C}_{+}$функция $\Theta$ (т.е. аналитическая и ограниченная в $\mathbb{C}_{+}$функция, граничные значения которой равны по модулю единище почти везде на $\mathbb{R}$ ) такая, что

$$
E=M_{\Theta} H_{0}, \quad M_{\Theta}=\mathscr{F}^{-1} \Theta \mathscr{F}
$$


где $\mathscr{F}$ и $\Theta$ есть преобразование Фурье и оператор умножения на функцию $\Theta$ соответственно. Согласно теореме Пэли-Винера $\mathscr{F} H_{0}=H^{2}\left(\mathbb{C}_{+}\right)$, где $H^{2}\left(\mathbb{C}_{+}\right)=H^{2}$ есть пространство Харди функций, аналитических в верхней полуплоскости и таких, что

$$
\|\eta\|^{2}=\sup _{y>0} \int_{\mathbb{R}}|\eta(x+i y)|^{2} d x<\infty .
$$

(Следует отметить, что мы отождествляем функции из класса Харди $H^{2}$ и их некасательные граничные значения на $\mathbb{R}$.) При этом, $\mathscr{F}\left(H_{0} \ominus E\right)=K_{\Theta}$, где $K_{\Theta}=H^{2} \ominus \Theta H^{2}$ есть так назьваемое модельное подпространство. Модельные подпространства играют исключительно важную роль в модели Секефальви-Надя-Фойаша для операторов сжатия [17], [18].

Заметим, что оператор $M_{\Theta}$ изометрически отображает пространство $H_{0}$ на пространство $E$. Следовательно, полугрупшы $S$ и $\left.S\right|_{E}$ унитарно эквивалентны, поскольку $M_{\Theta} S_{t}=\left.S_{t}\right|_{E} M_{\Theta}, t \geqslant 0$. В работах [2]-[4] была введена модель, описьвающая все марковские коциклы групшы $S$ в пространстве $H$ с точностью до унитарной эквивалентности. Пусть $U$ есть группа унитарных операторов в гильбертовом пространстве $K=H_{0} \ominus M_{\Theta} H_{0}$. Пара $(U, \Theta)$ определяет марковский унитарньй коцикл $W^{*}$ групшы $S$ по формуле

$$
\begin{gathered}
W_{t}^{*}=\left(U_{t} P_{K}+S_{t} P_{E}\right) S_{-t} P_{[t,+\infty)}+M_{\Theta} P_{[0, t]}+P_{H_{0}^{\perp}}, \\
W_{-t}^{*}=S_{-t} W_{t} S_{t}, \quad t \geqslant 0 .
\end{gathered}
$$

Здесь $P_{\mathscr{L}}, P_{[0, t]}$ и $P_{[t,+\infty)}$ обозначают ортогональные проекторы на подпространства $\mathscr{L}$ и подпространства функций с носителями на $[0, t]$ и $[t,+\infty)$ соответственно.

Формула (4) описьвает все марковские коциклы с точностью до унитарной эквивалентности. Рассмотрим группу унитарных операторов $\widetilde{S}=\left\{\widetilde{S}_{t}=W_{t}^{*} S_{t}, t \in \mathbb{R}\right\}$. Заметим, что сужение группы $\widetilde{S}$ на $H_{0}$ имеет вид $\widetilde{S}_{t}=\left.U_{t} \oplus S_{t}\right|_{E}, t \geqslant 0$. Как было указано выше, полугруппа $\left.S\right|_{E}$ унитарно эквивалентна полугруппе сдвигов $\left.S\right|_{H_{0}}$. Следовательно, группа $\widetilde{S}$ в пространстве $H$ унитарно эквивалентна групе $U \oplus S$ в пространстве $K \oplus H$. Поскольку по условию $\widetilde{S}_{t}=W_{t}^{*} S_{t}, t \in \mathbb{R}$, мы получаем $S_{t}=\underset{\widetilde{S}}{W_{t}} \widetilde{S}_{t}$, и семейство унитарных операторов $W$ является марковским коциклом группы $\widetilde{S}$. Рассмотрим $C_{0}$-полугруппу сжатий $T=\left\{T_{t}=\left.P_{K} S_{t}\right|_{K}, t \geqslant 0\right\}$ в пространстве $K$. Полугруппа $T$ определяет коцикл группы $U$ по формуле $L_{t}=T_{t} U_{-t}, t \geqslant 0$.

Теорема 2. Для коиикла $L=\left\{T_{t} U_{-t}, t \geqslant 0\right\}$, построенного по полугруппе "усеченных сдвигов" $T=\left\{T_{t}=\left.P_{K} S_{t}\right|_{K}, t \geqslant 0\right\} u$ группе унитарных операторов $U$, существует марковский унитарный коцикл $W=\left\{W_{t}, t \in \mathbb{R}\right\}$ группы $\widetilde{S}=U \oplus S$, являющийся минимальной дилатачией кочикла $L$, так что

$$
L_{t}=\left.P_{K} W_{t}\right|_{K}, \quad t \geqslant 0 \text {. }
$$

Явный вид дилатации дается формулой (4).

ДокАЗАТЕЛЬСТво. Определим действие группы $U$ на ортогональном дополнении к $K$ как тождественное и рассмотрим семейство унитарных операторов $A_{t}=W_{t} U_{t}, t \geqslant 0$. Согласно формуле (4) получаем

$$
A_{t}=S_{t} P_{K}+S_{t} P_{E} S_{-t} U_{t}+P_{[0, t]} M_{\Theta}^{*} U_{t}+P_{H_{0}^{\perp}}, \quad t \geqslant 0 .
$$


Из равенства (5) следует, что

$$
\left.A_{t}\right|_{K}=\left.S_{t}\right|_{K}+\left.P_{[0, t]} M_{\Theta}^{*} U_{t}\right|_{K}
$$

Покажем, что $\left.P_{[0, t]} M_{\Theta}^{*} U_{t}\right|_{K}=0$. Действительно, если $\eta \in K$, то $\mathscr{F} \eta=f \in K_{\Theta}$, а $M_{\Theta}^{*} \eta=\mathscr{F}^{-1} \bar{\Theta} f$. Хорошо известно (и немедленно вытекает из определения модельного пространства $K_{\Theta}$ ), что $\Theta \bar{f} \in K_{\Theta}$ для всякой функции $f \in K_{\Theta}$. Следовательно, $\bar{\Theta} f \in H_{-}^{2}=\left(H^{2}\right)^{\perp}$ и $\mathscr{F}^{-1} \bar{\Theta} f \in H_{0}^{\perp}$. Поэтому $P_{[0, t]} \mathscr{F}^{-1} \bar{\Theta} f=0$.

Итак, мы показали, что $\left.A_{t}\right|_{K}=\left.S_{t}\right|_{K}$. Следовательно,

$$
T_{t}=\left.P_{K} W_{t} U_{t}\right|_{K}, \quad t \geqslant 0,
$$

откуда немедленно следует доказьваемое утверждение.

3. Коциклические возмущения группы сдвигов на прямой. Рассмотрим коциклические возмущения $\widetilde{S}_{t}=W_{t} S_{t}, t \in \mathbb{R}$, группы сдвигов $S$ унитарными марковскими коциклами $W$. Из представления (4) следует, что сужение каждого такого возмущения $\widetilde{S}$ на подпространство $H_{0}$ имеет вид $\widetilde{S}_{t}=\left.U_{t} \oplus S_{t}\right|_{E}, t \geqslant 0$, где $E$ есть некоторое инвариантное подпространство полугруппы сдвигов в $H_{0}$, а $U$ - группа унитарных операторов в пространстве $K=E^{\perp}$. Ниже мы исследуем, какими свойствами должна обладать группа $U$, чтобы группа $\widetilde{S}$ являлась коциклическим возмущением группы $S$ коциклом $W$ со свойством $W_{t}-I \in \mathscr{I}_{2}$ при $t \in \mathbb{R}$, где $\mathscr{S}_{2}$ обозначает класс Гильберта-Шмидта.

Для любой группы $U$ унитарных операторов в гильбертовом пространстве $K$ найдется такое не более чем счетное семейство конечных мер $\mu_{n}$ на $\mathbb{R}, 1 \leqslant n \leqslant N \leqslant+\infty$, $\mu_{n}(\mathbb{R})<+\infty$, что группа $U$ будет унитарно эквивалентна групше унитарных операторов $\widetilde{U}$ в пространстве $\mathscr{H}=\bigoplus_{n=1}^{N} L^{2}\left(\mu_{n}\right)$, действующей по формуле

$$
\widetilde{U}_{t}\left(\bigoplus_{n=1}^{N} \eta_{n}\right)=\bigoplus_{n=1}^{N} U_{t}^{(n)} \eta_{n}
$$

где группа унитарных операторов $U_{t}^{(n)}$ действует в пространстве $L^{2}\left(\mu_{n}\right)$ так, что $\left(U_{t}^{(n)} \eta\right)(x)=e^{i t x} \eta(x)$. Представление (6) назьвается спектральным.

В статьях [3], [19] было показано, что в том случае, когда группа $U$ циклическая, а мера $\mu$, участвующая в спектральном представлении, сингулярна относительно меры Лебега, группа $\widetilde{S}$ есть коциклическое возмущение группы $S$ коциклом $W$ со свойством $W_{t}-I \in \mathscr{S}_{2}$ (соответствуюший результат был доказан в статье [3] для атомических мер и в [19] для непрерьвных сингулярных мер). Здесь мы распространим этот результат на случай счетной прямой суммы циклических групп.

ТЕОРема 3. Предположим, что меры $\mu_{n}$, участвующие в спектральном представлении группь $U$, сингулярны относительно меры Лебега. Тогда группа $\widetilde{S}=$ $U \oplus S$ является коциклическим возмущением группы, унитарно әквивалентной әруппе $S$, коииклом $W$ со свойством $W_{t}-I \in \mathscr{S}_{2}, t \in \mathbb{R}$.

Перед тем как доказьвать теорему 3, нам потребуется развить некоторую технику, которой посвящен следующий раздел. 
4. Теорема о триангуляции усеченного сдвига. Существенную роль в доказательстве теоремы 3 играет теорема П.Р. Ахерна и Д. Н. Кларка о триангуляции оператора усеченного сдвига (см. [21], а также [17, лекция V]). Как в статье [21], так и в [17] рассматриваются модельные подпространства для однократного сдвига в единичном круге. Нам же понадобится вариант теоремы о триангуляции для модельных подпространств полугруппы сдвигов в полуплоскости. Заметим, что операторы, составляющие полугруппу сдвигов, имеют бесконечную кратность, и классическую теорему о триангулящии нельзя применить автоматически. По этой причине нам необходимо привести полное доказательство соответствующих утверждений.

Мы начнем со случая непрерывной сингулярной меры. Пусть $\mu$ - непрерывная сингулярная мера на вешественной прямой $\mathbb{R}($ т.е. $\mu(\{t\})=0$ для всех $t \in \mathbb{R})$ и $\mu(\mathbb{R})<\infty$. Рассмотрим полугруппу унитарных операторов $U=\left(U_{t}\right)_{t \geqslant 0}$, действующих в пространстве $L^{2}(\mu)$ по формуле

$$
\left(U_{t} f\right)(x)=e^{i t x} f(x), \quad f \in L^{2}(\mu) .
$$

Определим сингулярную внутреннюю функцию $\varphi$ как

$$
\varphi(z)=\exp \left(i \int_{\mathbb{R}} \frac{d \mu(t)}{t-z}\right), \quad z \in \mathbb{C}_{+},
$$

и пусть $K=H_{0} \ominus M_{\varphi} H_{0}$. Отметим одно важное свойство внутренней функции $\varphi$, определенной формулой (7): как показано в [20], $\varphi-1 \in H^{2}$ и $\|\varphi-1\|_{2}^{2}=2 \mu(\mathbb{R})$.

ПРЕДЛОЖЕНИЕ 4. Полугруппа $U$ унитарно әквивалентна некоторой полугруппе $V=\left(V_{t}\right)_{t \geqslant 0}$, действующей в пространстве $K$ и такой, что $V_{t}=\left.P_{K} S_{t}\right|_{K}+R_{t}$, $t \geqslant 0$, әде $R_{t} \in \mathscr{S}_{2}$. При этом, $\left\|R_{t}\right\|_{\mathscr{S}_{2}}^{2} \leqslant C \mu(\mathbb{R})$, әде $C$ - некоторая константа, зависящая только от $t$.

ДокАЗАТЕльство. Преобразование Фурье унитарно отображает пространство $H_{0}$ на пространство Харди $H^{2}=H^{2}\left(\mathbb{C}_{+}\right)$, а подпространство $K$ на модельное подпространство $K_{\varphi}$. Отметим также, что $\mathscr{F} S_{t} \mathscr{F}^{-1}=E_{t}$, где полугруппа $\left(E_{t}\right)_{t \geqslant 0}$ действует в $H^{2}$ по формуле

$$
\left(E_{t} f\right)(x)=e^{i t x} f(x) .
$$

Таким образом, утверждение теоремы эквивалентно следующему утверждению.

Полугруппа $U=\left(U_{t}\right)_{t \geqslant 0}$ унитарно әквивалентна некоторой полугруппе $V=$ $\left(V_{t}\right)_{t \geqslant 0}$, действующей в пространстве $K_{\varphi}$ и такой, что $V_{t}=\left.P_{\varphi} E_{t}\right|_{K_{\varphi}}+R_{t}$, $t \geqslant 0$, где $P_{\varphi}-$ проектор в $H^{2}$ на модельное пространство $K_{\varphi}, R_{t} \in \mathscr{S}_{2} u$ $\left\|R_{t}\right\|_{\mathscr{S}_{2}}^{2} \leqslant C \mu(\mathbb{R})$.

Рассмотрим оператор, унитарно отображающий пространство $L^{2}(\mu)$ на модельное пространство $K_{\varphi}$. Для случая пространств на единичной окружности подобная конструкция приведена в [21] (см. также [17, лекция V]). Применяя конформное отображение круга на полуплоскость, мы получим аналогичньй результат для пространств на прямой и в полуплоскости.

Для $s \in \mathbb{R}$ обозначим через $\mu_{s}$ сужение меры $\mu$ на $(-\infty, s]$. Рассмотрим семейство сингулярных внутренних функций

$$
\varphi_{s}(z)=\exp \left(i \int_{\mathbb{R}} \frac{d \mu_{s}(t)}{t-z}\right), \quad z \in \mathbb{C}_{+} .
$$


Тогда оператор $\mathscr{V}$, определенный в пространстве $L^{2}(\mu)$ формулой

$$
(\mathscr{V} f)(z)=\sqrt{2} i \int_{\mathbb{R}} f(s) \varphi_{s}(z) \frac{d \mu(s)}{z-s}, \quad z \in \mathbb{C}_{+},
$$

осуществляет унитарное отображение пространства $L^{2}(\mu)$ на $K_{\varphi}$.

Мы имеем

$$
\begin{aligned}
\left(\mathscr{V} U_{t} f\right)(z) & =\sqrt{2} i \int_{\mathbb{R}} e^{i t s} f(s) \varphi_{s}(z) \frac{d \mu(s)}{z-s} \\
& =\sqrt{2} i e^{i t z} \int_{\mathbb{R}} f(s) \varphi_{s}(z) \frac{d \mu(s)}{z-s}-\sqrt{2} i \int_{\mathbb{R}} f(s) \varphi_{s}(z) \frac{e^{i t z}-e^{i t s}}{z-s} d \mu(s) \\
& =\left(E_{t} \mathscr{V} f\right)(z)+\left(J_{t} f\right)(z), \quad z \in \mathbb{C}_{+} .
\end{aligned}
$$

Покажем, что оператор $J_{t}$, действующий из $L^{2}(\mu)$ в $H^{2}$, принадлежит классу Гильберта-Шмидта и $\left\|J_{t}\right\|_{\mathscr{S}_{2}}^{2} \leqslant C \mu(\mathbb{R})$. Рассмотрим семейство операторов $J_{t}^{\varepsilon}: L^{2}(\mu) \rightarrow H^{2}$, $\varepsilon>0$, определенных как

$$
\left(J_{t}^{\varepsilon} f\right)(x)=\left(J_{t} f\right)(x+i \varepsilon)=-\sqrt{2} i \int_{\mathbb{R}} f(s) \varphi_{s}(x+i \varepsilon) \frac{e^{i t(x+i \varepsilon)}-e^{i t s}}{x+i \varepsilon-s} d \mu(s), \quad x \in \mathbb{R} .
$$

Поскольку $\|g(\cdot+i \varepsilon)\|_{H^{2}} \rightarrow\|g\|_{H^{2}}$ при $\varepsilon \rightarrow 0+$ для всякой функции $g \in H^{2}$, то $J_{t}^{\varepsilon} \rightarrow J_{t}$ в сильной операторной топологии. Покажем, что $J_{t}^{\varepsilon} \in \mathscr{S}_{2}$. Учитывая, что $\left|\varphi_{s}(z)\right| \leqslant 1$, $z \in \mathbb{C}_{+}, s \in \mathbb{R}$, получаем

$$
\left\|J_{t}^{\varepsilon}\right\|_{\mathscr{S}_{2}}^{2} \leqslant \int_{\mathbb{R}} \int_{\mathbb{R}}\left|\frac{e^{i t(x+i \varepsilon)}-e^{i t s}}{x+i \varepsilon-s}\right|^{2} d \mu(s) d x \leqslant C(t) \mu(\mathbb{R})
$$

так как

$$
\int_{\mathbb{R}}\left|\frac{e^{i t(x+i \varepsilon)}-e^{i t s}}{x+i \varepsilon-s}\right|^{2} d x \leqslant C(t)
$$

где константа $C(t)$ не зависит ни от $s$, ни от $\varepsilon>0$. Таким образом, для фиксированного $t \geqslant 0$ нормы Гильберта-Шмидта операторов $J_{t}^{\varepsilon}, \varepsilon>0$, равномерно ограничены. Переходя к сильному операторному пределу при $\varepsilon \rightarrow+0$, получаем, что $J_{t} \in \mathscr{S}_{2}$ и $\left\|J_{t}\right\|_{\mathscr{S}_{2}}^{2} \leqslant C(t) \mu(\mathbb{R})$.

Применяя к равенству (8) проектор $P_{\varphi}$ и оператор $\mathscr{V}^{*}$, получаем

$$
U_{t}=\mathscr{V}^{*} P_{\varphi} E_{t} \mathscr{V}+\mathscr{V}^{*} P_{\varphi} J_{t}
$$

что завершает доказательство предложения 4.

Теперь рассмотрим случай атомической меры $\mu$. Пусть

$$
\mu=\sum_{n=1}^{N} y_{n} \delta_{x_{n}}
$$

где $\delta_{x}$ обозначает меру Дирака (единичную нагрузку) в точке $x$, а $1 \leqslant N \leqslant \infty$. Мы считаем, что $\mu(\mathbb{R})<\infty$, т.е. $\sum_{n} y_{n}<\infty$. 
Положим $z_{n}=x_{n}+i y_{n}$ и рассмотрим произведение Бляшке

$$
B(z)=\prod_{n=1}^{N} \frac{z-z_{n}}{z-\bar{z}_{n}}, \quad z \in \mathbb{C}_{+} .
$$

Условие $\sum_{n} y_{n}<\infty$ гарантирует сходимость произведения (9). Более того, как показано в [20], произведение Бляшке $B$ обладает следующим важньм свойством: $B-1 \in H^{2}$ и $\|B-1\|_{2}^{2}=4 \pi \sum_{n} y_{n}=4 \pi \mu(\mathbb{R})$.

Будем отождествлять пространство $L^{2}(\mu)$ с пространством последовательностей $\left(f_{n}\right)_{n=1}^{N}$ таких, что $\sum_{n}\left|f_{n}\right|^{2} y_{n}<\infty$. Если полугрупша $U=\left(U_{t}\right)_{t \geqslant 0}$ действует в $L^{2}(\mu)$ по формуле $\left(U_{t} f\right)(x)=e^{i t x} f(x)$, то $U_{t}\left(\left(f_{n}\right)_{n=1}^{N}\right)=\left(e^{i t x_{n}} f_{n}\right)_{n=1}^{N}$. Пусть теперь $K=$ $H_{0} \ominus M_{B} H_{0}$.

ПРЕДЛОЖЕНИЕ 5. Полугруппа $U$ унитарно әквивалентна некоторой полугруппе $V=\left(V_{t}\right)_{t \geqslant 0}$, действующей в пространстве $K$ и такой, что $V_{t}=\left.P_{K} S_{t}\right|_{K}+R_{t}$, $t \geqslant 0$, где $R_{t} \in \mathscr{S}_{2}$. При этом, $\left\|R_{t}\right\|_{\mathscr{S}_{2}}^{2} \leqslant C \mu(\mathbb{R})$, где константа $C$ зависит только om $t$.

ДокАЗАТЕЛЬСТво. Согласно рассуждениям, приведенным в начале доказательства предложения 4 , нам достаточно показать, что полугруппа $U=\left(U_{t}\right)_{t \geqslant 0}$ унитарно эквивалентна некоторой полугруппе $V=\left(V_{t}\right)_{t \geqslant 0}$, действующей в модельном пространстве $K_{B}=H^{2} \ominus B H^{2}$ и такой, что

$$
V_{t}=\left.P_{B} E_{t}\right|_{K_{B}}+R_{t}, \quad t \geqslant 0
$$

где $P_{B}$ - проектор в $H^{2}$ на подпространство $K_{B}, R_{t} \in \mathscr{S}_{2}$ и $\left\|R_{t}\right\|_{\mathscr{S}_{2}}^{2} \leqslant C \mu(\mathbb{R})$.

В случае атомической меры $\mu$ построение оператора $\mathscr{V}$, унитарно отображающего $L^{2}(\mu)$ на $K_{B}$, оказьвается значительно проще, чем в случае непрерьвной сингулярной меры. Рассмотрим функции

$$
g_{n}(z)=\frac{1}{\sqrt{\pi}}\left(\prod_{m=1}^{n-1} \frac{z-z_{m}}{z-\bar{z}_{m}}\right) \frac{\sqrt{y_{n}}}{z-\bar{z}_{n}} .
$$

Хорошо известно (см. $\left[17\right.$, лекция V]), что функции $g_{n}$ образуют ортонормированный базис в $K_{B}$ (базис Мальмквиста-Уолша). Для $f=\left(f_{n}\right) \in L^{2}(\mu)$ положим

$$
\mathscr{V} f=\sum_{n=1}^{N} \sqrt{y_{n}} f_{n} g_{n}
$$

Очевидно, оператор $\mathscr{V}$ унитарно отображает $L^{2}(\mu)$ на $K_{B}$. Далее, имеем

$$
\begin{aligned}
\left(\mathscr{V} U_{t} f\right)(z) & =\sum_{n=1}^{N} \sqrt{y_{n}} f_{n} e^{i t x_{n}} g_{n}(z) \\
& =\sum_{n=1}^{N} \sqrt{y_{n}} f_{n} e^{i t z} g_{n}(z)+\sum_{n=1}^{N} \sqrt{y_{n}} f_{n}\left(e^{i t x_{n}}-e^{i t z}\right) g_{n}(z) \\
& =\left(E_{t} \mathscr{V} f\right)(z)+\left(J_{t} f\right)(z), \quad z \in \mathbb{C}_{+} .
\end{aligned}
$$


Покажем, что $J_{t} \in \mathscr{S}_{2}$ и $\left\|J_{t}\right\|_{\mathscr{S}_{2}}^{2} \leqslant C \mu(\mathbb{R})$. Прежде всего, заметим, что

$$
\left.\| e^{i t x_{n}}-e^{i t x}\right) g_{n} \|_{2}^{2}=\frac{y_{n}}{\pi} \int_{\mathbb{R}}\left|\frac{e^{i t x_{n}}-e^{i t x}}{x-x_{n}+i y_{n}}\right|^{2} d x \leqslant C(t) y_{n} .
$$

Рассмотрим действие оператора $J_{t}$ на ортонормированном базисе $f^{(n)}$ пространства $L^{2}(\mu)$, где $f_{k}^{(n)}=0, k \neq n$, и $f_{n}^{(n)}=\left(\sqrt{y_{n}}\right)^{-1}$. Тогда

$$
\left\|J_{t}\right\|_{\mathscr{S}_{2}}^{2}=\sum_{n}\left\|J_{t} f^{(n)}\right\|_{2}^{2}=\sum_{n}\left\|\left(e^{i t x_{n}}-e^{i t z}\right) \sqrt{y_{n}} g_{n}\right\|_{2}^{2} \leqslant C \sum_{n} y_{n} .
$$

Таким образом, предложение полностью доказано.

ЗАмЕчАниЕ. Название “теорема о триангулящи"” связано с тем, что в классическом случае оператора сдвига в классе Харди в круге оператор $R_{t}$ есть нижне-треугольный (вольтерров) оператор. Для нас, однако, существенны только включение $R_{t} \in \mathscr{S}_{2}$ и оценка нормы Гильберта-Шмидта оператора $R_{t}$.

Теперь мы объединим предложения 4 и 5 в одно утверждение, которым будем пользоваться при доказательстве теоремы 3 .

Пусть $\mu$ - сингулярная мера на вешественной прямой $\mathbb{R}$ и $\mu(\mathbb{R})<\infty$. Пусть полугруппа унитарных операторов $U=\left(U_{t}\right)_{t \geqslant 0}$ действует в пространстве $L^{2}(\mu)$ по формуле $\left(U_{t} f\right)(x)=e^{i t x} f(x)$.

Мы можем представить меру $\mu$ как сумму непрерьвной сингулярной и атомической мер, т.е. $\mu=\mu^{(1)}+\mu^{(2)}$, где мера $\mu^{(1)}$ не имеет точечных нагрузок, а $\mu^{(2)}=\sum_{n} y_{n} \delta_{x_{n}}-$ атомическая мера. Тогда пространство $L^{2}(\mu)$ изоморфно прямой сумме пространств $L^{2}\left(\mu^{(1)}\right)$ и $L^{2}\left(\mu^{(2)}\right)$, а полугруппа $U$ распадается в прямую сумму полугрупп: $U^{(1)} \oplus U^{(2)}$ $=\left(U_{t}^{(1)} \oplus U_{t}^{(2)}\right)_{t \geqslant 0}$, где

$$
\begin{array}{ll}
\left(U_{t}^{(1)} f\right)(x)=e^{i t x} f(x), & f \in L^{2}\left(\mu^{(1)}\right), \\
\left(U_{t}^{(2)} f\right)(x)=e^{i t x} f(x), & f \in L^{2}\left(\mu^{(2)}\right) .
\end{array}
$$

Определим сингулярную внутреннюю функцию $\varphi$ формулой $(7)\left(\right.$ с $\mu^{(1)}$ вместо $\left.\mu\right)$, а произведение Бляшке $B$ формулой (9). Положим

$$
\Theta(z)=\varphi(z) B(z)=\exp \left(i \int_{\mathbb{R}} \frac{d \mu^{(1)}(t)}{t-z}\right) \prod_{n=1}^{N} \frac{z-z_{n}}{z-\bar{z}_{n}}, \quad z \in \mathbb{C}_{+} .
$$

Функция $\Theta$ внутренняя, и $\Theta-1 \in H^{2}$ (см. [20]), причем

$$
\|\Theta-1\|_{2}^{2} \leqslant C \mu(\mathbb{R}) .
$$

Отметим следующее, хорошо известное свойство модельных пространств. Пусть $\Theta_{1}$ и $\Theta_{2}-$ внутренние функции. Тогда модельное пространство $K_{\Theta_{1} \Theta_{2}}$ представимо в виде ортогональной прямой суммы

$$
K_{\Theta_{1} \Theta_{2}}=K_{\Theta_{1}} \oplus \Theta_{1} K_{\Theta_{2}}
$$

В частности, $K_{\Theta}=K_{\varphi} \oplus \varphi K_{B}$, и проектор $P_{\Theta}$ на $K_{\Theta}$ имеет вид $P_{\Theta}=P_{\varphi}+\varphi P_{B} \bar{\varphi}$ (здесь $\varphi$ обозначает оператор умножения на функцию $\varphi$ ). Наконец, пусть $K=H_{0} \ominus M_{\Theta} H_{0}=$ $\mathscr{F}^{-1} K_{\Theta}$. 
Tеорема 6. Полугруппа $U$ унитарно әквивалентна некоторой полугруппе $V=$ $\left(V_{t}\right)_{t \geqslant 0}$, действуюшей в пространстве $K$ и такой, что $V_{t}=\left.P_{K} S_{t}\right|_{K}+R_{t}, t \geqslant 0$, əде $R_{t} \in \mathscr{S}_{2}$. При этом,

$$
\left\|R_{t}\right\|_{\mathscr{S}_{2}}^{2} \leqslant C \mu(\mathbb{R}), \quad t \in \mathbb{R},
$$

где $C$ - некоторая константа, зависящая только от $t$.

ДокАЗАТЕльство. Как обычно, мы перейдем к модельным пространствам в классе Харди с помощью преобразования Фурье. Нам надо построить такую полугруппу операторов $V=\left(V_{t}\right)_{t \geqslant 0}$ в пространстве $K_{\Theta}$, унитарно эквивалентную полугруппе $U=\left(U_{t}\right)_{t \geqslant 0}$, что

$$
V_{t}=\left.P_{\Theta} E_{t}\right|_{K_{\Theta}}+R_{t}, \quad t \geqslant 0,
$$

и $R_{t} \in \mathscr{S}_{2}$. Согласно предложениям 4 и 5 полугрупшы $U^{(1)}$ и $U^{(2)}$ унитарно эквивалентны полугруппам $V^{(1)}$ и $V^{(2)}$ в пространствах $K_{\varphi}$ и $K_{B}$ таким, что

$$
\begin{gathered}
V_{t}^{(1)}=\left.P_{\varphi} E_{t}\right|_{K_{\varphi}}+R_{t}^{(1)}, \quad t \geqslant 0, \\
V_{t}^{(2)}=\left.P_{B} E_{t}\right|_{K_{B}}+R_{t}^{(2)}, \quad t \geqslant 0 .
\end{gathered}
$$

При этом операторы $R_{t}^{(1)}$ и $R_{t}^{(2)}$ принадлежат классу Гильберта-Шмидта, $\left\|R_{t}^{(1)}\right\|_{\mathscr{S}_{2}}^{2} \leqslant$ $C \mu^{(1)}(\mathbb{R})$ и $\left\|R_{t}^{(2)}\right\|_{\mathscr{S}_{2}}^{2} \leqslant C \mu^{(2)}(\mathbb{R})$, где константа $C$ зависит только от $t$.

Определим полугруппу $V=\left(V_{t}\right)_{t \geqslant 0}$ следующим образом:

$$
V_{t}=V_{t}^{(1)} P_{\varphi}+\varphi V_{t}^{(2)} P_{B} \bar{\varphi}
$$

(напомним, что $\varphi$ обозначает оператор умножения на внутреннюю функцию $\varphi$ в пространстве $H^{2}$ ). В силу разложения $K_{\Theta}=K_{\varphi} \oplus \varphi K_{B} V$ есть полугруппа унитарных операторов в пространстве $K_{\Theta}$. Покажем, что вьполнено равенство (14). По определению полугруппы $V$

$$
V_{t}=P_{\varphi} E_{t} P_{\varphi}+\varphi P_{B} E_{t} P_{B} \bar{\varphi}+R_{t}^{(1)} P_{\varphi}+\varphi R_{t}^{(2)} P_{B} \bar{\varphi} .
$$

С другой стороны,

$$
\begin{aligned}
P_{\Theta} E_{t} P_{\Theta} & =\left(P_{\varphi}+\varphi P_{B} \bar{\varphi}\right) E_{t}\left(P_{\varphi}+\varphi P_{B} \bar{\varphi}\right) \\
& =P_{\varphi} E_{t} P_{\varphi}+\varphi P_{B} E_{t} P_{B} \bar{\varphi}+\varphi P_{B} \bar{\varphi} E_{t} P_{\varphi}
\end{aligned}
$$

так как оператор $P_{\varphi} E_{t} \varphi P_{B}$ тождественно равен нулю. Действительно, ясно, что операторы $E_{t}$ и $\varphi$ коммутируют, а $P_{\varphi}(\varphi f)=0, f \in H^{2}$. Наконец, заметим, что $P_{B} \bar{\varphi} P_{\varphi}=0$, поскольку $\bar{\varphi} f \in\left(H^{2}\right)^{\perp}$ для всякой функции $f \in K_{\varphi}$, и, значит, $P_{B} \bar{\varphi} f=0$. Следовательно,

$$
V_{t}=P_{\Theta} E_{t} P_{\Theta}+R_{t}^{(1)} P_{\varphi}+\varphi R_{t}^{(2)} P_{B} \bar{\varphi}+\varphi P_{B} \bar{\varphi}\left(P_{\varphi} E_{t}-E_{t} P_{\varphi}\right) .
$$

Теперь теорема будет доказана, как только мы покажем, что коммутатор $P_{\varphi} E_{t}-E_{t} P_{\varphi}$ принадлежит классу Гильберта-Шмидта и $\left\|P_{\varphi} E_{t}-E_{t} P_{\varphi}\right\|_{\mathscr{S}_{2}}^{2} \leqslant C \mu^{(1)}(\mathbb{R})$. Мы вынесем это утверждение в отдельное предложение (предложение 7 ), которое будет доказано в начале следующего раздела. 
5. Доказательство теоремы 3. Пусть $\mu$-конечная сингулярная борелевская мера на $\mathbb{R}$, а внутренняя функция $\Theta$ определена формулой (10). Напомним, что $K=$ $H_{0} \ominus M_{\Theta} H_{0}$, а $P_{K}$ обозначает проектор, действующий из $H_{0}$ на $K$. Нам понадобится следующее предложение о коммутаторе проектора $P_{K}$ и операторов сдвига $S_{t}$.

ПРеДЛОЖЕНИЕ 7. Имеем $P_{K} S_{t}-S_{t} P_{K} \in \mathscr{I}_{2} u\left\|P_{K} S_{t}-S_{t} P_{K}\right\|_{\mathscr{S}_{2}}^{2} \leqslant C \mu(\mathbb{R})$.

ДокАЗАТЕЛЬСТво. Преобразование Фурье устанавливает унитарную эквивалентность операторов $P_{K}$ и $S_{t}$ с операторами $P_{\Theta}$ и $E_{t}$, действующими в пространстве Харди $H^{2}$. Хорошо известно (см. [17]), что проектор $P_{\Theta}$ на подпространство $K_{\Theta}$ имеет вид

$$
\left(P_{\Theta} \eta\right)(z)=\frac{1}{2 \pi i} \int_{\mathbb{R}} \frac{1-\Theta(z) \overline{\Theta(s)}}{s-z} \eta(s) d s, \quad \eta \in H^{2}, \quad z \in \mathbb{C}_{+} .
$$

Чтобы обойти трудности, связанные с интерпретацией значений интеграла (15) при $z \in \mathbb{R}$, вновь воспользуемся приемом из доказательства предложения 4. Рассмотрим семейство операторов $Q_{\varepsilon}, \varepsilon>0$, действуюших в $H^{2}$ по формуле

$$
\left(Q_{\varepsilon} \eta\right)(x)=\left(P_{\Theta} \eta\right)(x+i \varepsilon)=\int_{\mathbb{R}} \frac{1-\Theta(x+i \varepsilon) \overline{\Theta(s)}}{s-x-i \varepsilon} \eta(s) d s, \quad \eta \in H^{2}, \quad x \in \mathbb{R} .
$$

Заметим, что $Q_{\varepsilon} \rightarrow P_{\Theta}$ в сильной операторной топологии при $\varepsilon \rightarrow+0$.

Ядро $D_{\varepsilon}(x, s)$ коммутатора $Q_{\varepsilon} E_{t}-E_{t} Q_{\varepsilon}$ имеет вид

$$
D_{\varepsilon}(x, s)=(1-\Theta(x+i \varepsilon) \overline{\Theta(s)}) \frac{e^{i t s}-e^{i t(x+i \varepsilon)}}{s-x-i \varepsilon} .
$$

Из условия $1-\Theta \in H^{2}$ немедленно вытекает, что $D_{\varepsilon} \in L^{2}(\mathbb{R} \times \mathbb{R})$, причем

$$
\left\|D_{\varepsilon}\right\|_{L^{2}(\mathbb{R} \times \mathbb{R})}^{2} \leqslant C\|1-\Theta\|_{L^{2}(\mathbb{R})}^{2} \leqslant 2 C \mu(\mathbb{R})
$$

с константой $C=C(t)$, не зависящей от $\varepsilon>0$. Таким образом, $Q_{\varepsilon} E_{t}-E_{t} Q_{\varepsilon} \in \mathscr{S}_{2}$, причем нормы Гильберта-Шмидта указанных операторов равномерно ограничены. Переходя к сильному операторному пределу при $\varepsilon \rightarrow+0$, получаем требуемую оценку для нормы Гильберта-Шмидта коммутатора $P_{\Theta} E_{t}-E_{t} P_{\Theta} \in \mathscr{S}_{2}$.

ДокАЗАТЕЛЬСТво тЕОРЕмЫ 3. Рассматриваемая группа $U$ унитарно эквивалентна группе $\widetilde{U}$, определенной формулой (6). С помощью замены масштаба мы всегда можем добиться того, что $\sum_{n} \mu_{n}(\mathbb{R})<\infty$. Обозначим через $\theta_{n}$ внутреннюю функцию, порожденную мерой $\mu_{n}$ согласно формуле (10), и положим $\Theta_{n}=\prod_{s=1}^{n} \theta_{s}$. Рассмотрим сингулярную меру $\mu=\sum_{n} \mu_{n}$. Пусть сингулярная внутренняя функция $\Theta$ определена равенством (10). Тогда $\Theta=\lim _{n \rightarrow+\infty} \Theta_{n}$ и $\Theta-1 \in H^{2}(\mathbb{R})$.

Рассмотрим подпространства

$$
L_{n}=H_{0} \ominus M_{\Theta_{n}} H_{0}, \quad K_{n}=M_{\Theta_{n-1}} L_{n}
$$

и положим

$$
K=\bigoplus_{n=1}^{N} K_{n}, \quad E=H_{0} \ominus K
$$


Поскольку $\Theta=\prod_{n=1}^{N} \theta_{n}$, из представления (12) следует, что $K=H_{0} \ominus M_{\Theta} H_{0}$.

По теореме 6 группа $\widetilde{U}$ унитарно эквивалентна групе $V_{t}=\bigoplus_{n=1}^{N} V_{t}^{(n)}$, где группа

$$
V_{t}^{(n)}=M_{\Theta_{n-1}} \widetilde{V}_{t}^{(n)} M_{\Theta_{n-1}}^{*} \mid K_{n}
$$

действует в пространстве $K_{n}$ и

$$
\widetilde{V}_{t}^{(n)}=\left.P_{L_{n}} S_{t}\right|_{L_{n}}+R_{t}^{(n)}, \quad t \geqslant 0
$$

При этом $R_{t}^{(n)} \in \mathscr{S}_{2}$.

Определим унитарные операторы $W_{t}$ с помощью формулы (4):

$$
W_{t}=\left(V_{t} P_{K}+S_{t} P_{E}\right) S_{-t} P_{[t,+\infty)}+M_{\Theta} P_{[0, t]}+P_{H_{0}^{\perp}}, \quad t \geqslant 0
$$

а для $t<0$ положим $W_{t}^{*}=S_{t} W_{-t} S_{-t}$. Тогда $W=\left(W_{t}\right)_{t \in \mathbb{R}}$ есть унитарный коцикл группы $S$, и группа $\widetilde{S}$ является коциклическим возмущением групшы $S$ коциклом $W$. Мы покажем, что $W_{t}-I \in \mathscr{S}_{2}$. Очевидно, достаточно проверить последнее включение для $t \geqslant 0$.

Имеем

$$
W_{t}-I=\left(V_{t}-S_{t}\right) P_{K} S_{-t} P_{[t, \infty)}+\left(M_{\Theta}-I\right) P_{[0, t]}, \quad t \geqslant 0 .
$$

Заметим, что

$$
\left.P_{K} S_{t}\right|_{K}=\left.\bigoplus_{n=1}^{N} M_{\Theta_{n-1}} P_{L_{n}} S_{t}\right|_{L_{n}} M_{\Theta_{n-1}}^{*}
$$

Следовательно,

$$
\left(V_{t}-S_{t}\right) P_{K}=\sum_{n=1}^{N} M_{\Theta_{n-1}}\left(V_{t}^{(n)}-\left.P_{L_{n}} S_{t}\right|_{L_{n}}\right) M_{\Theta_{n-1}}^{*}-P_{E} S_{t} P_{K}
$$

Согласно оценке (13)

$$
\left\|V_{t}^{(n)}-\left.P_{L_{n}} S_{t}\right|_{L_{n}}\right\|_{\mathscr{S}_{2}}^{2}=\left\|R_{t}^{(n)}\right\|_{\mathscr{S}_{2}}^{2} \leqslant C \mu_{n}(\mathbb{R}), \quad t \geqslant 0 .
$$

Следовательно,

$$
\left\|P_{K}\left(V_{t}-S_{t}\right) P_{K}\right\|_{\mathscr{S}_{2}}^{2} \leqslant C \sum_{n} \mu_{n}(\mathbb{R})<+\infty .
$$

Покажем, что $P_{E} S_{t} P_{K} \in \mathscr{S}_{2}$. Поскольку $E=H_{0} \ominus K$, имеем

$$
P_{E} S_{t} P_{K}=P_{E}\left(S_{t} P_{K}-P_{K} S_{t}\right) .
$$

Последний оператор принадлежит классу $\mathscr{S}_{2}$ согласно предложению 7.

Таким образом, мы показали, что оператор $\left(V_{t}-S_{t}\right) P_{K}$ (а, значит, и первое слагаемое в формуле (16)) принадлежит классу Гильберта-Шмидта $\mathscr{S}_{2}$. Оценим второе слагаемое в (16). Из условия $\Theta-1 \in L^{2}(\mathbb{R})$ вытекает, что оператор $\left(M_{\Theta}-I\right) P_{[0, t]}$ есть оператор Гильберта-Шмидта. Действительно, ядро оператора $(\Theta-1) \mathscr{F}: L^{2}([0, t]) \rightarrow H^{2}$ имеет вид

$$
k(x, y)=\frac{1}{\sqrt{2 \pi}}(\Theta(y)-1) e^{-i x y} .
$$

Следовательно, $k \in L^{2}([0, t] \times \mathbb{R})$, и, принимая во внимание неравенство $(11)$, получаем

$$
\left\|M_{\Theta}-I\right\|_{\mathscr{S}_{2}}^{2}=\|(1-\Theta) \mathscr{F}\|_{\mathscr{S}_{2}}^{2} \leqslant t\|\Theta-1\|_{2}^{2}=2 t \mu(\mathbb{R}) .
$$

Суммируя полученные результаты, находим, что $W_{t}-I \in \mathscr{S}_{2}, t \in \mathbb{R}$. 


\section{6. Коциклические возмущения колмогоровских потоков на гиперфинит-} ных факторах. В этом разделе мы используем сведения об алгебре канонических антикоммутационных соотношений, приведенные в приложении. Фиксируем оператор $R$, $0 \leqslant R \leqslant I$, в гильбертовом пространстве $H=L^{2}(\mathbb{R})$. Рассмотрим представление $\pi_{R}$ алгебры канонических антикоммутационных соотношений $\mathscr{A}(H)$ в пространстве $\mathscr{H}=$ $\Gamma(H) \otimes \Gamma(H)$, отвечающее состоянию $\omega_{R}$. Пусть семейство операторов $R_{t}, 0 \leqslant R_{t} \leqslant 1$, определено условием $R_{t} S_{t}=S_{t} R$. Группа сдвигов $S$ в пространстве $H$ определяет семейство инъективных *-гомоморфизмов $\tau_{t}$ алгебры $\pi_{R}(\mathscr{A}(H))$ на алгебру $\pi_{R_{t}}(\mathscr{A}(H))$ согласно следующей формуле:

$$
\tau_{t}\left(\pi_{R}(a(\eta))\right)=\pi_{R_{t}}\left(a\left(S_{t} \eta\right)\right), \quad \eta \in H, \quad t \in \mathbb{R}
$$

Пусть $\mathscr{M}_{R} \subset B(H)$ есть гиперфинитный фактор фон Неймана, порожденный алгеброй $\pi_{R}(\mathscr{A}(H))$. Предположим, что $R=P_{K}$ есть проектор на коинвариантное подпространство $K=H_{0} \ominus M_{\Theta} H_{0}$ полугруппы сдвигов, отвечающее внутренней функции $\Theta$.

ПРЕДЛОЖЕНИЕ 8. Пусть $\mu$ есть конечная сингулярная борелевская мера, а внутренняя функиия $\Theta$ определена формулой (10). Тогда *-гомоморфизм $\tau_{t}$ алгебры $\pi_{P_{K}}(\mathscr{A}(H))$ на алгебру $\pi_{S_{t} P_{K} S_{-t}}(\mathscr{A}(H))$ продолжается до *-гомоморфизма гиперфинитного фактора $\mathscr{M}_{P_{K}}$ на гиперфинитный фактор $\mathscr{M}_{S_{t} P_{K} S_{-t}}$.

ДокАЗАТЕЛЬСТво. Условие продолжения *-гомоморфизма $\alpha$ алгебры $\pi_{R}(\mathscr{A}(H))$, полученного квазисвободньм подъемом унитарного оператора $U$, до *-гомоморфизма гиперфинитного фактора $\mathscr{M}_{R}$ имеет вид $R U-U R \in \mathscr{S}_{2}$ (см. [22]). Таким образом, результат следует из предложения 7.

Семейство *-гомоморфизмов $\tau=\left\{\tau_{t}, t \in \mathbb{R}\right\}$ алгебры фон Неймана $\mathscr{M}_{0}$, образы которых принадлежат алгебре всех ограниченных операторов $B(\mathscr{H})$ в некотором фиксированном гильбертовом пространстве $\mathscr{H}$, естественно назвать колмогоровским потоком (см. $[23,3])$ на алгебре $\mathscr{M}=\bigcup_{t} \tau_{t}\left(\mathscr{M}_{0}\right)$ с уходящей алгеброй $\mathscr{M}_{0}$, если вьполнено условие

$$
\bigcap_{t} \mathscr{M}_{t}=\{\mathbb{C} 1\}
$$

где $\mathscr{M}_{t}=\tau_{t}\left(\mathscr{M}_{0}\right), t \in \mathbb{R}$, а символ 1 обозначает тождественный оператор в пространстве $\mathscr{H}$.

Пусть $\mathscr{M}$ и $\mathscr{M}_{0}$ суть гиперфинитные факторы фон Неймана, порожденные алгебрами $\pi_{P_{K}}(\mathscr{A}(H))$ и $\pi_{P_{K}}\left(\mathscr{A}\left(H_{0}\right)\right)$ соответственно. Положим $\mathscr{M}_{t}=\tau_{t}\left(\mathscr{M}_{0}\right)$. Здесь мы оставили то же самое обозначение для продолжения $\tau_{t}$ с алгебры $\pi_{P_{K}}(\mathscr{A}(H))$ на фактор $\mathscr{M}_{P_{K}}$, существующего в силу предложения 8.

ПРЕДЛОЖЕНИЕ 9. Семейство *-гомоморфизмов $\tau=\left\{\tau_{t}, t \in \mathbb{R}\right\}$ образует колмогоровский поток на алгебре $\mathscr{M}$ с уходящей алгеброй $\mathscr{M}_{0}$.

ДокАЗАТЕЛЬСТво. Можно записать факторы $\mathscr{M}_{t}$ в явном виде (см. приложение)

$$
\mathscr{M}_{0}=B(\Gamma(E) \otimes \Gamma(K)), \quad \mathscr{M}_{t}=B\left(\Gamma\left(S_{t} E\right) \otimes \Gamma\left(S_{t} K\right)\right)
$$

где $E \oplus K=H_{0}$, а $B(X)$ обозначает множество всех ограниченных операторов в гильбертовом пространстве $X$. Из равенства (17) немедленно следует, что $\bigcap_{t} \mathscr{M}_{t}=\{\mathbb{C} 1\}$. 
Пусть $W=\left\{W_{t}, t \in \mathbb{R}\right\}$ есть марковский коцикл грушшы сдвигов $S$. Рассмотрим коџиклическоевозмущение $\widetilde{S}=\left\{\widetilde{S}_{t}=W_{t} S_{t}, t \in \mathbb{R}\right\}$ групшы $S$. Положим $P_{t}=S_{t} P_{K} S_{-t}$ и $\widetilde{P}_{t}=W_{t} P_{t} W_{t}^{*}, t \in \mathbb{R}$. Пусть $w_{t}$ есть $*$-гомоморфизм алгебры $\pi_{P_{t}}(\mathscr{A}(H))$ на алгебру $\pi_{\widetilde{P}_{t}}(\mathscr{A}(H))$. Введем обозначение $\widetilde{\mathscr{M}}_{t}=\mathscr{M}_{\widetilde{P}_{t}}$.

Теорема 10. Пусть марковский коиикл $W$ удовлетворяет условию $W_{t}-I \in \mathscr{S}_{2}$. Тогда *-гомоморфизм $w_{t}$ продолжсается до *-гомоморфизма гиперфинитного фактора $\mathscr{M}_{t}$ на гиперфинитный фактор $\widetilde{\mathscr{M}}_{t}$, так что получающееся семейство *-гомоморфизмов $w_{t} \circ \tau_{t}$ образует колмогоровский поток на гиперфинитном факторе $B(\Gamma(H \ominus K) \otimes\{\mathbb{C} \Omega\})$ с уходящей алгеброй $B(\Gamma(E) \otimes\{\mathbb{C} \Omega\}) \subset \widetilde{\mathscr{M}}_{0}$.

ДокаЗАТЕЛьСтво. Из условия $W_{t}-I \in \mathscr{S}_{2}$ вытекает, что $P_{t} W_{t}-W_{t} P_{t} \in \mathscr{I}_{2}$. Следовательно, согласно [22] *-гомоморфизм $w_{t}$ продолжается с $\pi_{P_{t}}(\mathscr{A}(H))$ на $\mathscr{M}_{t}$. Заметим, что

$$
\widetilde{\mathscr{M}}_{t}=w_{t}\left(\mathscr{M}_{t}\right)=B\left(\Gamma\left(\widetilde{S}_{t} E\right) \otimes \Gamma(K)\right)
$$

Следовательно, $\mathcal{N} \equiv B(\Gamma(E) \otimes\{\mathbb{C} \Omega\}) \subset \widetilde{\mathscr{M}}_{0}$, и определено сужение $\tau_{t}$ на $\mathcal{N} . \mathrm{C}$ другой стороны, $\bigcap_{t} \tau_{t}(\mathscr{N})=\{\mathbb{C} 1\}$.

Приложение. Алгебра канонических антикоммутационных соотношений и ее представления. Ниже приведены сведения из работ [22], [24].

Пусть $H$ есть сепарабельноегильбертово пространство. Алгеброй канонических антикоммутационны х соотношений $\mathscr{A}(H)$ назьвается алгебра с инволюцией *, единицей 1 и образующими $a(\xi), a^{*}(\eta), \xi, \eta \in H$, удовлетворяюшими соотношениям:

1. $a^{*}(\alpha \xi+\beta \eta)=\alpha a^{*}(\xi)+\beta a^{*}(\eta), \alpha, \beta \in \mathbb{C}$

2. $a(\xi) a^{*}(\eta)+a^{*}(\eta) a(\xi)=(\eta, \xi) \mathbf{1}$

3. $a(\xi) a(\eta)+a(\eta) a(\xi)=0$.

Любой оператор $R, 0 \leqslant R \leqslant I$, определяет состояние (линейньй положительный функционал) $\omega_{R}$ на алгебре $\mathscr{A}(H)$ по формуле

$$
\omega_{R}\left(a^{*}\left(\eta_{1}\right) \cdots a^{*}\left(\eta_{n}\right) a\left(\xi_{1}\right) \cdots a\left(\xi_{m}\right)\right)=\delta_{n m} \operatorname{det}\left(\left(R \eta_{i}, \xi_{j}\right)\right) .
$$

Состояние (18) называется квазисвободным.

Антисимметричным произведением $n$ копий пространства $H$ называется гильбертово пространство $\Lambda^{n} H$, образованное линейной оболочкой векторов $\eta_{1} \Lambda \eta_{2} \Lambda \cdots \Lambda \eta_{n}$, $\eta_{i} \in H$, со скалярньм произведением

$$
\left(\eta_{1} \Lambda \cdots \Lambda \eta_{n}, \xi_{1} \Lambda \cdots \Lambda \xi_{n}\right)=\operatorname{det}\left(\left(\eta_{i}, \xi_{j}\right)\right)
$$

Антисимметричным пространством Фока над $H$ называется гильбертово пространство $\Gamma(H)=\{\mathbb{C} \Omega\} \oplus\left(\bigoplus_{n=1}^{+\infty} \Lambda^{n} H\right)$. Здесь первое слагаемое в суммепредставляет собой одномерное пространство, натянутое на вектор $\Omega$, назьваемый вакуумньм.

Будем предполагать, что алгебра $\mathscr{A}(H)$ реализована в качестве подалгебры алгебры ограниченных операторов в $\Gamma(H)$. При этом, действие образующих $\mathscr{A}(H)$ задается формулой $a^{*}(\eta) \xi_{1} \Lambda \cdots \Lambda \xi_{n}=\eta \Lambda \xi_{1} \Lambda \cdots \Lambda \xi_{n}$, и $a(\eta)$ является сопряженным с $a^{*}(\eta)$. Заметим, что при этом $\|a(\eta)\|=\left\|a^{*}(\eta)\right\|=\|\eta\|_{H}$, и $\mathscr{A}(H)$ становится $C^{*}$-алгеброй относительно операторной нормы в $\Gamma(H)$. 
Другие представления алгебры $\mathscr{A}(H)$ могут быть получены с помощью конструкции Гельфанда-Наймарка-Сигала по различным состояниям на $\mathscr{A}(H)$. Квазисвободное состояние (18) определяет представление $\pi_{R}$ алгебры $\mathscr{A}(H)$ в пространстве $\mathscr{H}=$ $\Gamma(H) \otimes \Gamma(H)$. Фиксируем ортонормированньй базис $\left(\eta_{n}\right)_{n=1}^{+\infty}$ в $H$ и оператор $J$ в $\Gamma(H)$, определяемый условиями $J \Omega=\Omega, J a(\eta)=-a(\eta) J$. Тогда отображение $\pi_{R}$, заданное формулой

$$
\pi_{R}\left(a\left(\eta_{n}\right)\right)=a^{*}\left((I-R)^{1 / 2} \eta_{n}\right) \otimes J+I \otimes a\left(R^{1 / 2} \eta_{n}\right),
$$

можно продолжить по линейности, так что получится представление алгебры $\mathscr{A}(H)$, при котором $\omega_{R}\left(x^{*} y\right)=\left(\pi_{R}(x), \pi_{R}(y)\right)_{\Gamma(H) \otimes \Gamma(H)}, x, y \in \mathscr{A}(H)$. Алгебра фон Неймана $\mathscr{M}_{R}=\pi_{R}(\mathscr{A}(H))^{\prime \prime}$, где $X^{\prime}$ обозначает коммутант множества $X$, является фактором, т.е. $\mathscr{M}_{R} \cap \mathscr{M}_{R}^{\prime}=\{\mathbb{C} 1\}$. $\Phi$ актор $\mathscr{M}_{R}$ назьвается гиперфинитным фактором фон Неймана или просто гиперфинитным фактором.

Пусть сжимающий оператор $T$ действует в гильбертовом пространстве $H$. Тогда *-отображение $\alpha_{T, R}$, заданное на образующих $\mathscr{A}(H)$ формулой $\alpha_{T, R}(a(\eta))=a(T \eta)$, $\eta \in H$, может быть единственным образом продолжено до вполне положительного отображения $C^{*}$-алгебры $\mathscr{A}$, переводящего состояние $\omega_{R}$ в состояние $\omega_{R^{\prime}}$, определяемое условием $R^{\prime} T=T R$, так что $\omega_{R^{\prime}}\left(\alpha_{T, R}(x)\right)=\omega_{R}(x), x \in \mathscr{A}(H)$. В том случае, когда оператор $T$ унитарен, отображение $\alpha_{T, R}$ является $*$-автоморфизмом алгебры $\mathscr{A}(H)$. В этом случае положим $\tau_{U, R}\left(\pi_{R}(x)\right)=\pi_{R^{\prime}}\left(\alpha_{U, R}(x)\right), x \in \mathscr{A}(H)$. Тогда $\tau_{U, R}$ есть инъективньй *-гомоморфизм алгебры $\pi_{R}(\mathscr{A}(H))$ на алгебру $\pi_{R^{\prime}}(\mathscr{A}(H))$. Говорят, что отображения $\alpha_{T, R}$ и $\tau_{U, R}$ заданы квазисвободным подъемом оператора $T$.

\section{СПИСОК ЦИТИРОВАННОЙ ЛИТЕРАТУРЫ}

[1] Amosov G. G. Cocycle perturbation of quasifree algebraic $K$-flow leads to required asymptotic dynamics of associated completely positive semigroup // Infin. Dimen. Anal. Quantum Probab. Rel. Top. 2000. V. 3. № 2. P. 237-246.

[2] Amosov G. G. Stationary quantum stochastic processes from cohomological point of view // Quantum Probability and Infinite Dimensional Analysis (Burg, 2001) / ed. W. Freudenberg. Quantum Probab. White Noise Anal. V. 15. River Edge, NJ: World Sci. Publ., 2003. P. 29-40.

[3] Amosov G. G. On Markovian cocycle perturbations in classical and quantum probability // Intern. J. Math. Math. Sci. 2003. № 54. P. 3443-3467.

[4] Амосов Г. Г. О марковских возмущениях группы унитарных операторов, ассоциированной со случайным процессом со стационарньми приращениями // Теор. вероятн. и ее прим. 2004. T. 49 . C. $145-155$.

[5] Accardi L., Frigerio A., Lewis J. T. Quantum stochastic processes // Publ. Res. Inst. Math. Sci. 1982. V. 18. №1. P. 97-133.

[6] Parthasarathy K. R. An Introduction to Quantum Stochastic Calculus. Monographs in Mathematics. V. 85. Basel: Birkhäuser Verlag, 1992.

[7] Lindsay J.M., Wills S. J. Markovian cocycles on operator algebras adapted to a Fock filtration // J. Funct. Anal. 2000. V. 178. P. 269-300.

[8] Liebscher V. How to generate Markovian cocycles on boson Fock space // Infin. Dimen. Anal. Quantum Probab. Rel. Top. 2001. V. 4. P. 215-219.

[9] Bhat B. V.R. Minimal isometric dilations of operator cocycles // Integral Equations Operator Theory. 2002. V. 42. № 2. P. 125-141.

[10] Amosov G. G. On cocycle conjugacy of quasifree endomorphism semigroups on the CAR algebra // J. Math. Sci. 2001. V. 105. P. 2496-2503.

[11] Булинский А. В. Алгебраические $K$-потоки и полупотоки сдвигов Пауэрса // УМН. 1996. T. 51 . C. $145-146$.

[12] Powers R. T. An index theory for semigroups of *-endomorphisms of $\mathscr{B}(\mathscr{H})$ and types $\mathrm{II}_{1}$ factors // Canad. J. Math. 1988. V. 40. №1. P. 86-114. 
[13] Arveson W. Continuous analogues of Fock space // Mem. Amer. Math. Soc. 1989. V. 80. № 409. P. 1-66.

[14] Bhat B. V.R., Skeide M. Tensor product systems of Hilbert modules and dilations of completely positive semigroups // Infin. Dimen. Anal. Quantum Probab. Rel. Top. 2000. V. 3. P. 519-575.

[15] Barreto S. D., Bhat B. V.R., Liebscher V., Skeide M. Type I product systems of Hilbert modules // J. Funct. Anal. 2004. V. 212. P. 121-181.

[16] Accardi L., Amosov G. G., Franz U. Second quantized automorphisms of the renormalized square of white noise (RSWN) algebra // Infin. Dimen. Anal. Quantum Probab. Rel. Top. 2004. V. 7. P. 183-194.

[17] Никольский Н. К. Лекции об операторе сдвига. М.: Наука, 1980.

[18] Секефальви-Надь Б., Фойаш Ч. Гармонический анализ операторов в гильбертовом пространстве. М.: Мир, 1970.

[19] Amosov G. G., Baranov A. D. On perturbations of the group of shifts on the line by unitary cocycles // Proc. Amer. Math. Soc. 2004. V. 132. P. 3269-3273.

[20] Баранов А. Д. Изометрические вложения пространств $K_{\Theta}$ в верхней полуплоскости // Проблемы матем. анализа. 2000. Т. 21. С. 30-44.

[21] Ahern P. R., Clark D. N. On functions orthogonal to invariant subspaces // Acta Math. 1970. V. 124. P. 191-204.

[22] Araki H. On quasifree states of CAR and Bogoliubov automorphisms // Contemp. Math. 1985. V. 62. P. 21-141.

[23] Emch G. G. Generalized K-flows // Commun. Math. Phys. 1976. V. 49. P. 191-215.

[24] Evans D. Completely positive quasifree maps on the CAR algebra // Commun. Math. Phys. 1979. V. 70. P. 53-68.

(Г.Г. Амосов) Московский физико-технический институт

Поступило

(А. Д. Баранов) Санкт-Петербургский государственный университет

02.02 .2005

E-mail: amosov@fizteh.ru, d.baranov@mail.ioffe.ru 